\title{
DIFFICULTÉS DANS L'APPRENTISSAGE DU MESURAGE
}

\author{
LARCHER, C. ${ }^{1}$, SÉRÉ, M.G. ${ }^{2}$ y JOURNEAUX, R. ${ }^{2}$ \\ ${ }^{1}$ L.I.R.E.S.T. Groupe de Didactique des Sciences de Paris 7. \\ ${ }^{2}$ DidaScO, Université de Paris XI.
}

\section{SUMMARY}

This paper presents the difficulties encountered by students of first University year, when carrying out measurements in order to answer a question concerning the isochronism of oscillations of a simple pendulum. It is based on a case study which concerns students who followed a course about measurement veforehand. The global strategies of these students are described. Then the difficulties encountered are analysed. We distinguish two categories: firstly, the difficulties stepping from relationships between a theoretical approach of modelised objects and an experimental approach of given objects; secondly the difficulties coming from the conceptualisation of differences between the measured values and the reference value. Eventually, elements of the response built by the students are analysed, particularly by assessing the reinvestment of the notions of statistics studied beforehand.

Le mesurage est une démarche fondamentale dans l'activité des scientifiques. Cependant peu d'informations sont đisponibles sur l'apprentissage de cette démarche ou sur les đifficultés đes élèves à la mettre en ouvre. En effet les situations expérimentales qui servent de support aux questionnements dans les recherches portant sur l'utilisation par les élèves de relations entre grandeurs physiques sont souvent des situations décrites déjà modélisées. Certains travaux se sont interessés aux démarches de construction de relations fonctionnelles entre grandeurs à partir d'activités expérimentales (Kuhn et ał. 1988, Johsua Dupin,1989, Lemeignan Weil-Barais 1989). Cependant les activités de mesurage et de traitement de la dispersion des mesures sont évacuées soit en restant dans le domaine qualitatif, soit en ayant recours à des simulations sur ordinateur.

Cette absence est à mettre en relation avec les pratiques habituelles dans les classes. Bien que les programmes français envisagent un traitement statistique des mesures dès la classe de seconde (programme 1987), il est peu courant de voir pratiquer en classe des activités de mesurage. Dans les séances de travaux pratiques proposées au lycée ou en début d'université, un protocole expérimental détailié est en général imposé aux élèves sans toujours être justifié par rapport à un problème posé - et il est rarement demandé de répéter les mesures plus d'une fois. L'incertitude sur une mesure est évaluée par un calcul classique qui somme toutes les incertitudes recensées, procédure qui est comprise par les étudiants comme une détermination et non pas comme une évaluation.
Conscients d $\mathrm{t}:$ Ia carence que comporte un enseignement expérimental où la réflexion critique sur les mesures est absente, nous avons mis en place en première année d'université an enseignement centré sur le mesurage visant à faire acquérir la notion de "confiance que l'on peut accorder au résultat d'un mesurage» avec un point de vue statistique (Bevington 1969). Cet enseignement a comporté un cours théorique sur les erreurs et incertitudes de mesure ainsi que sur le traitement statistique des mesures. Plu:iieurs travaux sont disponibles pour construire un tel er.seignement; citons McFarland et al. (1987), Reed 1989, Piccard (1989) et Peterson (1991). Le cours a été suivi de «leux séances de travaux pratiques d'environ quatre heure.s. Lors de ces séances, les étudiants ont effectué des séries de mesures d'une même grandeur, mis en commun leurs résultats, puis utilisé des relations statistiques four calculer le taux de confiance lié au résultat obterıu; ils ont aussi commenté leurs résultats en termes de précision et d'exactitude. Le but de ces expérimentations était explicitement la mesures: mesurer la distance focale d'une lentille par différentes méthodes, puis évaluer la résistivité d'un alliage en mesurant la résistance, le diamètre et la longueur d'un fil de cet alliage. Vu le: temps consacré aux mesures elles-mêmes et aux traitements statistiques, il n'était prévu aucune utilisation des mesures pour caractériser l'écart entre une théorie $\epsilon t$ l'expérience ou pour valider un modèle. L'intérêt d'z.méliorer la précision et l'exactitude des mesures pour répondre à đe telles questions avait été évoqué par l'enseignant mais aucune activité pratique n'avait été mise en ceuvre avec cette finalité. L'observation de ces séances de Travaux Pratiques et le recueil des 
productions des étudiants ont permis une analyse des difficultés rencontrées par les étudiants dans ces activités (Séré M.G., Larcher J., Journeaux R. 1992).

L'objectif de l'étude présentée ici est d'analyser les démarches d'étudiants, ayant suivi l'enseignement décrit ci-dessus, lorsqu' on leur propose de se prononcer sur I'isochronisme des oscillations d'un pendule pesant à partir de mesures de durées d'oscillations. A cette fin, des entretiens semi-directifs ont été menés avec quatre étudiants trois mois après cet enseignement. II s'agit d'une étlide de cas; parmi les quatre étudiants, deux ont été interrogés séparément, ils sont noté $\mathrm{S}$ et $\mathrm{A}$; detux ont été interrogés ensemble, ce binôme est noté HL. Dans un premier temps nous décrirons la démarche des étudiants observés, en référence à une analyse de la tâche présentée préalablement. Dans un deuxième temps, nous analyserons les difficultés rencontrées par les élèves; nous distinguerons d'une part les difficultés liées aux relations que les étudiants cherchent à établir entre leurs connaissances théoriques antérieures et la question particulière qui leur est posée et d'autre part les difficultés liées à l'utilisation des concepts permettant de situer les différentes mesures par rapport à la valeur cherchée. Nous analyserons ensuite les savoirs, méthodes et critères qu'ils mettent en cuvre pour répondre à la question posée et nous essaierons d'évaluer leurs acquisitions concernant le traitement statistique des mesures. Ces analyses tiennent compte des connatissances que nous avons obtenues par ailleurs (observations de classe) sur les conceptualisations que font les étuđiants de ce niveau à propos đu mesurage.

\section{Présentation et analyse de la tâche}

La tâche proposée aux élèves lors de ces entretiens est de déterminer la valeur de la période d'un pendule pesant lorsqu'il est écarté de sa position d'équilibre d'un petit

Figura 1

Dispositif expérimental.

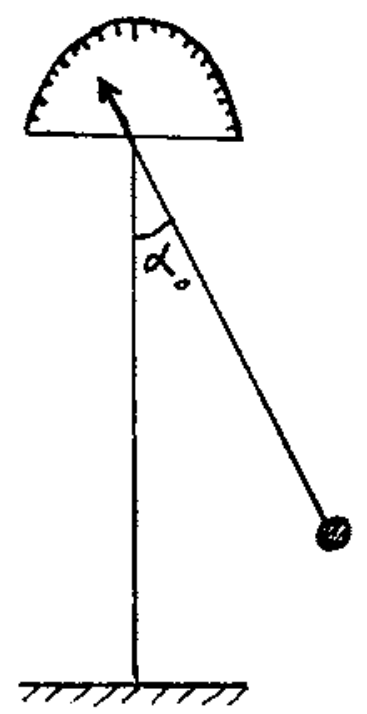

angle et de juger si la période est la même pour un grand angle.

Il s'agit d'un pendule monté sur un roulement à bille, I'axe du pendule est prolongé vers le haut par un index qui se déplace devant un disque građué de degré en degré permettant de repérer les angles $\alpha_{0}$ (Fig. 1).

Au cours de leur scolarité les étudiants interrogés avaient étudié le pendule élastique. Le programme indiquait «équation différentielle du mouvement d'un oscillateur harmonique non amorti - fréquence propre - conservation de I'énergie - amortissement d'un oscillateur». Certains manuels prévoyaient une différenciation avec le pendule pesant prototype d'un oscillateur périodique mais non harmonique. A propos de l'amortissement d'un oscillateur élastique les manuels proposaient une mise en évidence expérimentale de la diminution de l'amplitude en fontion du temps associée à un isochronisme des oscillations.

Notre étude préalable de ce pendule a comporté tune évaluation de l'amortissement: en 10 oscillations il perd 4 degrés à partir d'une position initiale faisant un angle $\alpha_{0}=30^{\circ}$ par rapport à la verticale et il perd 7 degrés à partir d'une position initiale faisant un angle $\alpha_{0}=60^{\circ}$ par rapport à la verticale.

Figura 2

Variation de la durée $\theta$ d'une oscillation en fonction de l'angle $\alpha$, initial, La plage d'amortissement est indiquée à partir de $\alpha_{0}=30^{\circ} \mathrm{et}$ $d e=60^{\circ}$. Chaque + correspond à une valeur moyenne obtenue sur 10 oscillations consécutives.

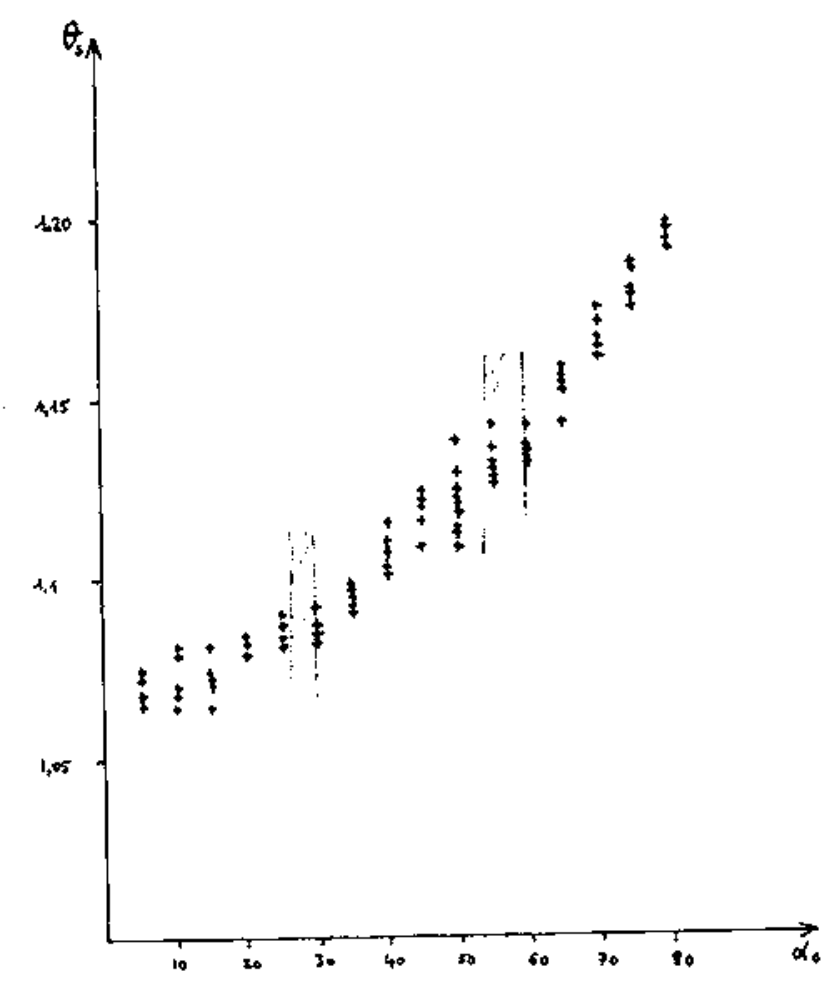


Les mesures ont été effectuées avec un chronomètre au $1 / 100$ déclenché lorsque le pendule passe par la verticale. L'ensemble des mesures que nous avons effectuées sur ce pendule est reporté fig. 2 . Il permet de constater que, compte tenu de la dispersion des mesures, de la variation avec l'angle de la durée d'une oscillation et de l'amortissement, on peut évaluer la période comme un dixième de la durée de dix oscillations.

La précision obtenue conduit à affirmer la différence entre la période du pendule pour un petit angle $\left(\alpha_{0}=10^{\circ}\right)$ et sa période pour un grand angle $\left(\alpha_{0}=60^{\circ}\right)$.

\section{DESCRIPTION DE LA DÉMARCHE DE MESURAGE DES ETUDIANTS}

Deux chronomètres sont mis à la disposition des élèves; pour le remier, une division représente $1 / 100$ ème de seconde, pour le second elle représente 1/10ème de seconde. Les étudiants interrogés ont utilisé, avec beaucoup de difficultés d'ailleurs, le chronomètre au $1 / 100$ mais sans argumenter ce choix de matériel.

Figura 3

Valeurs de $\theta$ obtenues par les étudiants pour différents angles $\alpha$ Plusicurs symboles côte à côte repèrent des vateurs de $\theta$ obtenues plusicurs fois pour un méme angle. codage: $\mathrm{S} \triangle \mathrm{A}+\mathrm{HL}$ *
L'ensemble des mesures de chaque étudiant est reporté graphiquement fig. 3. Cette figure donne une idée de la dispersion de leurs mesures.

\section{Mode opératoire}

Dans les trois entretiens le déclenchement du chronomètre s'est fait à l'instant où l'angle entre le pendule et la verticale est maximum. La vitesse est alors considérée comme nulle sauf pour $\$$ qui doute de la reproductibilité des conditions de lancer: ( $̧$ a dépend) comment on lance le pendule.

La méthode consistant à déclencher le chronomètre lorsqu'il passe par la verticale a été proposée à Setà H.L; cette méthođe a été rejetée. Pour $\mathrm{S}$ le temps sera plus court mais on n'appréciera pas forcemment au bon moment tandi:i que là (en haut) le temps sera plus long donc on a plus de chances d'appuyer au bon moment puis à l'ail ia ça se voit mieux quand le pendule redescend que quand il passe. Pour H.L quand puis-je considérer qu'il est à la verticale? je sais pas il va trop vite et puis si je prend la mesure ici il faut que je sache quand est-ce cu'il est à la verticale et dans quel sens il $v a$, deux fois de suite dans le temps il passe à la verticale et il y en a un que je dois éliminer. Ainsi il vaut mieux pour les élève:s avoir le temps de déclencher le chronomètre. En transposant cette mesure de durée à une mesure de distance, cela revient à préférer repérer une large tache plutôt qu'un point.

\section{Prise en compte de l'amortissement}

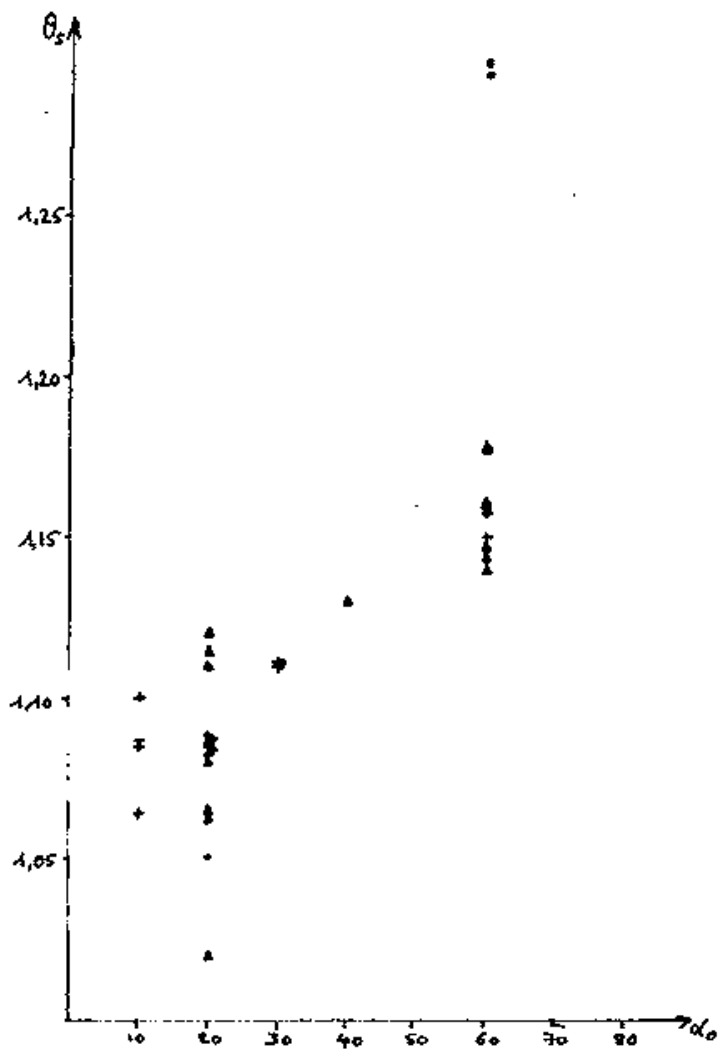

Si l'amortissement est envisagé, la mesure est faite sur un seul ou sur un nombre pas trop grand d'aller-retour:

S: si je compte 4 aller-retour ça sera moins bon que sion compte un seul je suppose - ça s'amortit - même si c'est négligeable - il vaut mieux faire un aller-retour probablement

A: ce que je pense faire c'est calculer pour plusieurs oscillations ce sera plus précis - il va y avoir des frottements - on peut pas en faire beaucoup - on va en faire 10 par exemple.

L'amortissement peut aussì être jugé non perceptible ce qui fait moye:mer $\mathrm{HL}$ sur 20 oscillations: parce que fa va se conserver à peu près normalement la graduation, elle va toujours donner $20^{\circ}$.

Ces décisions ont été prises a priori sans essai préalable.

\section{Multiplicité et critique des mesures}

Le niveau d'analyse critique des mesures est assez différent suivant liss étudiants.

A commence par faire plusieurs mesures pour une osciIlation pour th angle initial de $10^{\circ}$ et commente ses difficultés je bouge aussi - ça va pas être précis - ça va 
pas être sur le $10^{\circ}$ exactement c'est pas très précis parce que j'appuie sur le chrono quand je lache l'appareil et c'est pas facile.

HL proposent d'emblée de mesurer la durée de 20 oscillations on va faire 20 oscillations, 20 aller-retour avec $20^{\circ}$. Puis ils analysent les causes d'incertitude. Le chrononètre est arrêté normalement au moment où il a terminé son retour mais il y a une grande incertitudeil y a une' incertitude au départ et il y en a une à l'arrivée - pour appuyer sur le chrono - le temps de réaction - il y a au moins une seconde-j'ai arrêté quand j'ai estimé qu'il était arrêté ici-mais déjà au début du décrochement et à l'arrêt il y a le temps de réaction qui va jouer - et puis on voit que ça s'arrête mais on n'est pas complètement sur que c'est arrivé jusqu'en haut.

Par contre $S$ fait une mesure pour un aller-retour en ayant lâché le pendule d'un angle de $20^{\circ}$ et arrêté le chronomètre quand le pendule revient en haut de sa course après une oscillation «ça fait 1 seconde $11 / 100$ ». Elle propose alors après cette unique mesure de voir si c'est la même chose pour un angle plus grand que $20^{\circ}$. Elle fait alors une mesure pour $\alpha=60^{\circ}$ et obtient ls $28 / 100$ puis une mesure pour $\alpha_{0}=40^{\circ}$ et obtient Is $13 / 100$. Elle commente alors ses trois valeurs T20,T40 et T60 doncle temps n'augmente pas avec l'angle, c'est pas proportionnel-, mais on peut peut être considérer que c'est le même temps - je sais pas si $13 / 100$ et $11 / 100$ on peut dire que c'est la même chose - je pense qu'il faudrait faire plein de mesures et voir si toutes les mesures se trouvent dans cette zone-là. Ainsi, la variation obtenue, qui n'est pas une fonction linéaire de l'angle, incite $S$ à se poser la question de la fiabilité des valeurs obtenues. Comparant alors $\mathrm{T} 20$ et $\mathrm{T} 40$ dont les valeurs sont proches, elle $s$ 'interroge sur la précision de ses mesures et envisage des causes possibles d'imprécision qui permettraient d'expliquer qu'on obtienne deux valeurs différentes au lieu de deux valeurs identiques parce que en fait c'est vraiment relatif - l'ombre (ombre de l'index sur le disque de repérage des angles) je la vois mais elle est floue - c'est pas vraiment sur zéro (quand le pendule est arrêté).

Le choix du nombre de mesures qu'on va effectuer n' est pas sounis à validation compte tenu du problème posé mais relève plutôt d'un dogme affirmé mais pas forcément pratiqué ce qu'il faudrait faire c'est une dizaine de mesures-dixmesures c'est peut-être pas assez s' interrogent H.L.; je pense qu'il faut toujours faire beaucoup de mesures - oui, c'est un peu léger 4 ou 5 affirme $S$ qui pourtant avait commencé par donner une réponse en se basant sur une seule mesure.

Par contre le fait de trouver deux valeurs identiques pour deux mesures successives donne une confiance dans cette valeur telle qu'il n'est pas besoin de multiplier les mesures. A la ciuestion tu as fais deux mesures, hein? suggérant que cen'est peut être suffisant, A. répondoui, mais jetrouvais pareil pour les deux, presque pareil; puis plein de bonne volonté je peux recommencer, mais je pense que ça va confirmer cette façon de raisonner est peut être induite par les pratiques habituelles en travaux pratiques où la deuxième mesure n'est là que pour confirmer la première.

\section{Moyens proposés pour améliorer les mesures}

Pour $\mathrm{HL}$, il vaut mieux que ce soit toujours le même qui lâche et qui déclenche; pour A au contraire il faut que je change, par exemple partir de là (de l'autre côté) et je le tiendrai d'une autre manière. Par ailleurs HL et A proposent d'automatiser le mesurage:

A: il faudrait qu'il y ait un appareil qui tienne le pendule et qu'en appuyant sur un bouton ça ferait lâcher et déclencherait le chrono

HL:il faudrait une cellule photoélectrique fixe et à chaque passage devant elle compte à ce moment-là.

\section{ANALYSE DES DIFFICULTÉS RENCON- TREES PAR LES ETUDIANTS}

\section{Confrontation théorie-expérience}

«Une observation, c'est une interprétation: c'cst intégrer une certaine vision dans la représentation théorique quı l'on se fait de la réalité (Fourez 1988).

L'importance de l'épistémologie de l'élève sur la ma. nière dont ils abordent l'enseignement des sciences physiques a été notée par différents auteurs (Gil \& Carrascosa 1985, Larochelle \& Desautels 1991) Pour les étudiants interrogés ici, les connaissances très formelles dont ils disposent sur les mouvements périodiques viennent brouiller la représentation de la tâche qui leur est proposée.

Notons tout d'abord que la tâche proposée étonne S la période, pour des petites oscillations et pour des grandes, et il faut pas que je fasse des calculs? quelle représentation du rôle de l'expérience ont donc les étudiants?

H.L et $S$ essaient avant toute mesure de prévoir la réponse à la question en faisant appel à leurs connaissances théoriques:

H.L ont tendance à modéliser le pendule en minimisant l'effet des frottements pour pouvoir raisonner sur le pendule parfait: oui je pense - comme le système est conservateur - là il n'y est pas complètement parce que au niveau de l'axe ce rotation - au bout d'un moment ça va s'amortir - mais si on prend un temps suffisamment court on peut dire qu'il est conservateur et l'amortissement sera négligeable, l'amplitude des oscillations - et je pense que la période ne change pas parce que l'énergie que j'ai mis au départ - enfin c'est vrai qu'il est amorti mais en tout cas pour les petites oscillations l'amortissement il est quand nême petit.

Pour $\mathrm{S}$ la prévision est plus difficile la période c'est un temps pour un angle donné, quelque soit l'angle, le temps sera le même. La période, moi je vois ça sur une courbe en fait c'est le temps entre deux points là - un motif - ça n'a rien à voir avec l'amplitude de la courbe doncl'amplitude des oscillations, il me semble que quand il fait de grandes oscillations il va plus vite pour un même temps donné - la vitesse va être différente mais je 
ne sais pas si ça a un rapport avec l'angle - c'est que même si le temps est le même, l'angle n'est pas le même mais elle prévoit quand même mon idée tout de suite c'est de dire non, mais je sais pas - automatiquement l'angle est plus grand mais peut être que le pendule va plus vite que pour des petits angles.

A n'a pas fait de prevision avant les mesures mais il essaie de justifier la différence obtenue expérimentalement je pense qu'on doit trouver la même chose pourles 10 périodes quel que soit l'angle mais si le système était parfait - il y a une différence parce que il y a des frottements comme le temps est plus long pour un angle de $30^{\circ}$ que pour un angle de $20^{\circ}$ (constatation) parce que plus l'angle est grand plus les frottements sont grands, c'est l'explication de la différence mais il s'interroge ensuite sur la définition de la période du pendule c'est la valeur considérée quand il n'y a pas de frottement, pour un appareil parfait? $j$ 'arrive pas à comprendre parce que la période change avec l'angle la période c'est sa qualité propre quoi quand il est parfait quand il n'est pas soumis à des frottements

Par ailleurs il semble que pour Iui le terme exact qualifie la valeur théorique de la période du pendule supposé parfait et non pas la valeur, que l'on cherche à cerner par les mesures, de la durée d'une oscillation di pendule réel pour un angle donné. L'amortissement est perçu comme cause de variation de la période avec l'angle et non pas comme cause de la variation de l'angle, le pendule parfait devrait «posséder une période quelle que soit l'amplitude des oscillations je considère une valeur proche de la valeur exacte - il faudrait éliminer les frottements; ces frottements gênent effectivement les étudiants pour utiliser le terme période et ils lui préfèrent oscillation ou aller retour.

\section{DIFFÉRENCIATION DE CONCEPTS}

\section{Exactitude/précision. Incertitude/erreur}

Ces termes sont souvent mal différenciés. A. avoue d'ailleurs je confonds toujours précis et exact, j'ai jamais su faire la différence; $\mathrm{S}$. fournit sa définition de la précision C'est déjà plus précis - ca veut pas dire exact, fa veut dire qu'on a enlevé tout ce qui pouvait fausser le résultat - il reste toujours quelque chose elle avait introđuit le terme précis après qu'on l'ait incitée à se mettre debout devant la graduation pour faire sa mesure ah out, c'est plus précis parce que je vois mieux.

A, comparant ses mesures pour une oscillation et pour dix oscillations, dit c'est ce que j'avais avec 10 oscillations, c'est surement ces mesures qui sont les plus précises -j'ai le choix entre précis et exact? c'est celle qui se rapproche le plus de la valeur exacte out c'est à la fois plus précis parceque quand je fais 10 oscillatio$n s .$. heu c'est pas évident je ne sais pas comment dire pour un petit angle c'est pas facile de compter une période par contre si j'en chronomètre dix c'est plus précis et donc plus exact et il reprend plus tard ah voilà ça y est je comprends je suis d'une part plus précis et étant donné que j'ai pris un temps plus long avec 10 oscillations 'ai éliminé des erreurs d'observation, de réflexe-je les limitées - ça veut dire que les erreurs de réflexe ont nioins d'effet

Pour H.L on n'aura jamais une valeur exacte-d'abord pour avoir une valeur exacte peut - être il faudrait faire un calcul - il y aura toujours une incertitude - tout ce qu'on peut jaire c'est d'essayer d'améliorer la précision - on peut toujours améliorer la qualité au moment où on mesure là-haut, au moment où on déclenche le chrono, mais savoir si le résultat est exact il faudrait je sais pas. Pour eux la précision est bien reliée à la dispersion; four voir si la méthode qui consiste à fixer le disque gradié plutôt que l'index mobile est plus précise ils proposen: ce qu'il faudrait faire c'est une dizaine de mesures contme tout à l'heure tu prends le même angle pour qu'on puisse comparer - l'intervalle entre les mesures va ́tre plus petit - sur 10 mesures on devrait se rendre compte de ça-10 mesures c'est peut être pas assez quand même.

\section{Aléatoire/systématique}

S. perçoit qu'il y a des erreurs qu'on peut éviter déjà voir si j'ai pu rerdre la mesure plus fausse-peut-être que je suis mal organisée pour prendre la mesure et elle les qualifie bien de systématiques tout à l'heure je vous ai dit carrément j'ai appuyé trop tôt c'était vraiment une erreur systénatique - peut-être j'ai tendance à appuyer avant mais c'est pas systématique - tout à l'heure quand j'étais assise et je lisais de là c'était systématique.

H.L précisent à propos de l'erreur faite en déclenchant le chrono elles sont aléatoires bien sûr-elles sont systématiques dans le sens que je les fais à chaque fois mais à chaque fois elles sont différentes.

Pour A. ce 'rocabulaire n'est pas encore acquis ce sera plus précis varce que en une seconde il peut se passer beaucoup c'e choses c'est un temps très court si je mesure 10 ascillations ça va être plus long ce sera une manière de Itmiter l'erreur... heu quels types d'erreur il y a? (réporse systématique et aléatoire) oui l'erreur systématique - les deux en fait - aléatoire je pense que ça limitera l'erreur aléatoire puis il éprouve le besoin de préciser le sens de ces termes aléatoire c'est quand on n'est pas responsable une erreur qui arrive... et systématique ça dépend de moi; lorsqu'on Iui donne une autre caractérisation de ces termes: aléatoire: dans un sens ou dans l'autre, systématique: toujours dans le même sens et toujours, il modifie son jugement sur le fait de limiter Ies erreurs en comptabilisant 10 oscillations alors ça dépend des deux mais sans argumenter. Plus tard il évite d'utiliser I'un ou I'autre terme pour éliminer les incertitudes humaines, ne sachant probablement pas si l'erreur qu'il introduit est toujours dans le même sens ou pas; it aurait fallu plutôt introduire le caractère de constance quantitative de l'erreur systématique. 


\section{Incertitude absolue/incertitude relative}

Pour A. il apparaît clairement que la détermination de la durée d'une oscillation sera plus précise à partir de la mesure de 10 oscillations ce sera une manière de limiter l'erreur par contre pour $\mathrm{S}$. cela ne semble pas du tout évident le problème sera toujours le même-quand estce que je vais déclencher le chrono et quand est-ce que je vais l'arrêter, mais je suis sûre d'avoir au moins à l'intérieur de ces deux aller retour un aller-retour qui sera impeccable - mais je vois pas pourquoi ce seraplus précis puis après quelques essais qui ne lui paraissent pas convaincants on trouve pas que les mêmes valeurs, il y a toujours l'écart qui est assez grand - en fait ça change rien - ça ne dépend pas du nombre d'allerretour qu'il fait parce qu'ily a toujours le même problème de lecture - il y aura toujours le début et la fin qui sera...

Lorsqu'on lui rappelle que ce sera le début et la fin d'un seul aller-retour qui seront litigieux elle commence aे relativiser l'incertitude ah oui, ce sera sur un temps plus grand et sur ce temps plus grand on va enlever $1 / 4$ de seconde alors que si on enlève $1 / 4$ de seconde sur un temps plus petit... donc il vaut mieux faire 10 aller retour; comme le temps va être plus grand, même si je rajoute ou j'enlève un ou deux centièmes de seconde ça se verra moins - il y aura plus de chiffres qui seront les mêmes clle oublie alors les problèmes d'amortissement qui lui faisaient đire un peu plus tôt en faisant deux allerretourça va être encore pire parce qu' il y al'amortissement qui va entrer en jeu. Il est bien difficile de gérer plusieurs problèmes à la fois?

Pour le binôme H.L les avis sont partagés plus on prend d'oscillations et plus la mesure de la période sera bonne je pense -- entre 20 et 10 oscillations ce qui va se passer c'est que l'incertitude va être beaucoup moindre parce que le temps que je réagisse s'oppose à non, f̧a va être plus long puisque à chaque mesure tu as une incertitude qui s'ajoute - à chaque période tu vas avoir une incertitude ce qui oblige le premier étudiant à expliciter son point de vue non, je veux dire tu comptes tes périodes approximativement et l'incertitude c'est au départ et à l'arrivée-je veux dire on a la première qui est litigieuse et la dernière qui est litigieuse par contre on a 18 mesures qui sont exactes parce que tu n'interviens pas dessus - u les laisses - si il y en a une seule qui est litigieuse au début et à la fin l'incertitude sera plus petite même si le terme incertitude relative n'est pas prononcé, il s'agit bien de cela; il ajoute d'ailleurs si on calcule sur 100 ou 200 oscillations l'erreur du début et l'erreur de la fin sera divisée par autant de fois qu'on a d'oscillations exactes entre les deux donc elles seront de plus en plus négligeables, par contre si on en prend une seule, comme c'est la première et la dernière qui sont mawaises...! Notons au passage que le qualificatif négligeable est ici aussi confondu avec petit; $S$ disait aussi fá s'amortit - même si c'est négligeable - il vaut mieux faire un aller-retour probablement l' aspect volontariste de négliger une grandeur devant une autre n'apparait pas.

\section{EXPLOITATION DES MESURES POUR REPONDRE A LA QUESTION}

\section{Construction de la moyenne}

Pour A. et S. I'usage de la moyenne nécessite des contraintes de nombre de valeurs. Pour A on peut faire la moyenne mais là ce serait pas forcément exact - parce qu'il y a pas assez de valeurs mais à un autre moment, faisant la moyenne de ses 5 mesures pour $10^{\circ}$ il dit j'aurais donné Is 05 - à peu près parce qu'il y a cinq mesures, ça peut pas être précis le nombre de valeurs influe donc sur l'exactitude et sur la précision. Pour S. je pense que je ferais la moyenne - faudrait faire plus de mesures mais elle introduit une nouvelle contrainte si elles avaient été plus proches, je pense que j'aurais fait une moyenne, mais là quand même! de $1 \mathrm{~s} 6.5 / 100$ à $1 \mathrm{~s} 121$ 100 il y a trop d'écart je trouve. Rappelons que $\mathrm{S}$ discernait mal l'aspect relatif de l'incertitude.

Dans les trois cas les étudiants on fait la moyenne des mesures effectuées avec la même méthode, celle jugée la meilleure, en écartant délibérément les valeurs antérieures ça ne peut que perturber dit A. et S. non je ne les mélange pas - fa n'a aucun sens de mélanger des valeurs sur un aller-retour et sur 10 aller-retourl'inceritude est beaucoup plus grande notons que si elle semble maintenant convaincue que la mesure sur 10 aller retour est meilleure que celle sur un aller retour, le vocabulaire utilisé ne s'est pas précisé, l'incertitude n'est pas encore relative, ce qui risque de l'induire en erreur lorsqu'elle sera à nouveau confrontée à ce problème.H.L proposent aussi de faire la moyenne en excluant les mesures là à partir du moment où on a compté sur les graduations.

\section{Formalisation du résultat - sa signification - signification de $\mathrm{I}^{+}$encadrement}

Le nombre de chiffres significatifs n'est pas toujours porteur d'information: ainsì A. fait de tête une moyenne et đit $j$ 'aurais donné l seconde 05 à peu près parce qu'il y a cinq mesures, ça peut pas être précis.

Interrogé sur le nombre de chiffres significatifs qu'il donne A. dit d'abord $I$ seconde et puis trois chiffres, j'irais jusqu'au millième puis en observant le chronomètre qu'il a utilisé il constate qu'il peut faire la différence entre $82 / 100$ et $83 / 100$, donc j'iraisjusqu'à deux. Parcontre lorsqu'on lui fait remarquer qu'en faisant la moyenne sur 10 oscillationss il a donné 1 seconde $15.8 / 100$ il répond qu'en faisant la moyenne il gagne en précision et qu'il peut donner 3 chiffres pour les centièmes.

En ce qui concerne la signification du résultat et en particulier de la place de la valeur cherchée: $\$$ si on fait la moyenne on trouve peut être jamais cette valeur mais elle sera dans le tas si on met un intervalle - plus ou moins quelque chose peut - elle être ailleurs? oui c'est encore possible mais alors c'est qu'on a fait toutes nos mesures avec des valeurs fausses cette expression pas très académique semble bien traduire une notion correcte de ce qu'est la justesse; de même pour A. la valeur exacte 
elle devrait se trouver si il n'y avait aucune erreur commise-elle serait comprise puis l'écartentre ces deux extrêmes ce serait mon incertitude absolue, je peux pas trouver en dehors de la moyenne plus ou moins cette incertitude, je prends un risque! - non on peut pas tomber en dehors cet intervalle ce serait pour Iut les valeurs les plus extrêmes que j'ai trouvé pour chaque angle. II dit ensuite je peux pas affirmer que la valeur exacte soit comprise dans ces bornes - si mon incertitude absolue est très grande je suis sûr, mais c'est pas intéressant, il faudrait trouver l'incertitude absolue la plus petite possible et celle qui soit jamais dépassée.

Pour H.L le résultat devra être donné sous la forme de une moyenne avec un intervalle de confiance - en précisant le nombre de chances que j'ai de trouver dans cet intervalle.

Pour S, ce plus ou moins quelque chose signifie que la période se trouve entre mais si on lui demande si elle peut le garantjr bah non justement c'est pour ça que j'avais une autre idé, faire comme on a fait en physique, en statistiques, prendre $90 \%$ de chances de tomber dans cet intervalle; elle envisage de calculer cet intervalle toutes les incertitudes qu'on peut calculer - je sais pas sic'est vraiment calculable dont par ailleurs elle perçoit mal l'utilisation: si vous voulez calculer quelque chose de précis à partir de la période il va falloir que vous calculiez pour toutes les périodes comprises dans cet intervalle. Notons aussi que pour $S$ cette incertitude qui đépend fortement du manipulateur tout dépend de la personne n'est donc pas une information utile à transmetre il faudrait faire vous même cet intervalle sur ce que vous allez trouver.

\section{Quels sont les critères pour juger si une différence est significative}

La réponse à cette question a été donnée plus ou moins tôt dans l'entretien suivant les cas, c'est-à-dire en particulier soit après discussion sur les aspects statistiques (cas de S.) soit avant (cas de A. et H.L).

Pour S. qui répond à cette question après s'être représenté l'ensemble des mesures qu'on pourrait faire sous forme d'un histogramme pour un angle donné, la comparaison des courbes va permettre de juger on va faire la même chose et si on obtient la même courbe pas décalée si on peut superposer les deux courbes ou bien si les intervalles de confiance qu'on a fixé se chevauchent elle essaie alors d'imaginer deux courbes je sais pas, je doute, si on regarde juste le temps si on trace la courbe là, si on superpose à l'autre ce sera décalé alors c'est pas la même période - mais pourtant il y en a qui vont être superposés; finalement, pour juger la période identique il faudrait dit-elle qu'elles soient complètement superposées - soit que ça fasse au moins un plateau, qu'il n'y ait pas deux bosses, pas de creux. La notion de taux de confiance n'est pas réinvestie.

H.L. raisonnent globalement sur l'ensemble des mesures sur les écarts on peut dire que la période augmente parce que là, en excluant les mesures là, à partir du moment où ont a compté sur les graduations, c'étaient des valeurs quitétaient quand même assez proches, alors que là on trouve 25 - je crois pas qu'on ait fait d'erreur - peut être il, 'aut voir sur un peu plus de mesures mais ça apparaît sîrement que c'est différent puis ils proposent d'essayer de voir sur les moyennes, oui j'aurais calculé les moyennes et comparé. Bien qu'ayant parlé d'intervalle de confiance auparavant ils ne mobilisent pas cette notion pour comparer les valeurs.

A. commenct: par comparer les valeurs qu'il a obtenu pour des angles de $10^{\circ}, 20^{\circ}$ et $30^{\circ}$ : en utilisant la même méthode des 10 oscillations pour un angle de $20^{\circ}$ et un angle de $30^{\circ}$ on a trouvé un résultat un peu plus grand pour l'angle de $30^{\circ}$ et étant donné que c'est la même méthode - ve: qu'on a recommencé deux fois la même chose et il y $x$ une différence puis il compare avec ses mesures à $61^{\circ}$ il y a un écart d'une seconde entre un angle de $20^{\circ}$ 't un angle de $60^{\circ}$ et même en tenant compte des imprécisions fa devrait pas varier comme ça; son raisonnemenl: prend en compte la đispersion ou plutôt la non dispersion de ses valeurs, la validité des mesures étant assurée par je trouvais presque pareil pour les deux.

\section{QUEL RÉINVESTISSEMENT DES NOTIONS DE STATISTIQUES VUES PREALABLEMENT?}

H.L semblent avoir acquis un vocabulaire adéquat sur les aspects statistiques l'intervalle entre les mesures va être plus pelit - sur 10 mesures on devrait se rendre compte de ç', sur l'écart type on va voir ça la moyenne avec un intervalle de confiance, mais cela fonctionne-til? en particulier ne confond-il pas l'étendue des mesures et l'écart-tyre?

Pour $\mathrm{S}$. ces termes restent bien vagues à en juger par le dialogue suivant on avait fait un intervalle de confiance et puis on avait dit on a $90 \%$ de chances dans cet intervalle mais là, je sais pas comment établir ce \% comment on I'avait établi en physique? je sais plus, siavec des écarts - types tout f̧a... et ça représentait quoi? ah ça je sais pas, on devrait faire comme tout à l'heure, les valeurs ciui sont plus ou moins loin de la moyennevous pouvez savoir combien vous avez de valeurs - en fait on calcile un chiffre et on sait combien on a de valeurs de chaque coté de la moyenne, non? c'est pas calculable sa? priée de s'expliquer davantage sur la procédure à suivre elle précise: après un chiffre qui donne un renseignement - sur comment vont s'étaler toutes les valeurs, où se trouve la moyenne là-dedans et alors après on prend un \% et on élimine les valeurs qui sont de chaque côté. (avec 100 mesures) on en aurait eu beaucoup de regroupées autour de la moyenne et puis quel. ques unes à thaque extrémité - ça aurait fait une courbe mais déjà il faut choisir un \% c'est ça que je comprends pas, si on ait $95 \%$ par exemple on va choisir dans la courbe, on 'ra juste enlever les extrémités et si on veut $40 \%$ on va retirer, on va aller de plus en plus vers le sommet, on \%a rétrécirla surface bah oui! il semble donc qu'elle ait réussi à se construire une représentation du taux de confiance. 
Par ailleurs, ellc raisonne en faisant appel au hasard $b a h$, on peut être tombé sur une valeur loin à propos d'une valeur marginale obtenue dans une série de mesures - mais cettc valeur sera ensuite éliminée, non considérée pour faire la moyenne par exemple. Elle s'inquiète pourtant de la validité de son jugement on sait pas sic'est le $6 / 100$ qui est juste ou toutes les autres peuvent être fausses bah je sais pas, on peut faire plein de mesures et on verrait bien; la répétition des mesures est bien envisagée pour pallier les effets du hasard.

Pour A qui a oublié le vocabulaire employé en statistique, son raisonnement le conduit à il faudrait trouver l'incertitude absolue la plus petite possible et celle qui soit jamais dépassée quand on lui rappelle le mot fiabilité, sa réaction est bah voilà, c'est fa qui me manquait donc là je pourrai dire dans l'incertitude absolue que j'ai trouvée je peux parler de confiance à tant pour cent je suis súr que la période sera comprise dans cet intervalle-l'incertitude absolue, ca ne veut pas dire que je trouverai pas une mesure en dehors - il faut que je dise à combitn, quelle est la confiance que j'accorde à cet intervalle - il faut l'intervalle le plus petit possible et la confiance la plus grande possible alors il faut que j'arrive à cerner. Ainsi bien que gêné par le qualificatif «absolu» perçu comme limitatif, il arrive Iui aussi à saisir le principe de l'intervalle de confiance.

\section{CONCI,USION}

Par rapport à l'ensemble des travaux que nous avons menés à propos du traitement statistique des mesures, ces entretiens mettent en lumière les démarches individuelles d'étudiants devant une tâche de mesurage, les raisonnements qu'ils développent ainsi que les ressources cognitives dont ils disposent. En dépit de différences d'un entretien à l'autre, il est possible de dégager quelques caractéristiques communes aux quatre étudiants interviewés et représentatives d'un ensemble plus large d'étudiants.

Les étudiants ont montré peu d'enthousiasme pour recommencer leurs mesures malgré une profession de foi souvent exprimée mais au conditionnel «il faudrait faire beaucoup de mesures». Ils s'attendent d'ailleurs peu à obtenir une dispersion. Face à cettc dispersion ils réagissent comme l'ont fait d'autres étudiants avant et pendant l'enseignement, ils arrêtent leur recueil de mesures lorsque deux résultats sont identiques ou sélectionnent parmi les mesures celles qu'ils vont faire entrer dans la moyenne. La moyenne n'a pas pris pour eux le statut de meilleur représentant d'une série de mesures.

Ils ont eu beaucoup d'idées pour détecter des causes de dispersion des mesures. Ils sont capables de minimiser une cause de dispersion - parfois au détriment de l'exactitude de la mesure - mais ils ont des difficultés à analyser et à comparer l'effet de différentes causcs de dispersion afin d'élaborer un protocole expérimental optimal. Ainsi le protocole expérimental qu'ils ont proposé pour déterminer la durée d'unc oscillation a été peu questionné. L'amortissement est envisagé mais non étudié; aucun moyen de contrôle de la validité de leur hypothèse sur l'importance de cet amortissement n'est proposé. La démarche consistant à moyenner sur plusieurs oscillations pour déterminer la durée d'une oscillation pour un angle donné est proposée ou rejetée mais n'est pas considérée comme une hypothèse qu'on peut analyser et valider; ils en restent à des affirmations.

L'étude de ce pendule s'est heurtée par ailleurs aux connaissances théoriques des étudiants sur le pandule: tout se passe comme s'ils étaient enfermés dans la théorie d'un pendule harmonique. Ils sont alors impuissants à imaginer une procédure reposant sur l'essai et la mesure pour évaluer l'influence de I'amortissement sur la détermination de la période du pendule pour un angle $\alpha_{\mathrm{o}}$ donné.

Les problèmes rencontrés par les étudiants leur ont permis de préciser les concepts présentés en cours, les notions d'intervalle de confiance et de taux de confiance en particulier qui sont apparus nécessaires pour essayer de répondre à la question posée: la période du pendule est-elle la même pour un petit angle et pour un grand angle?

Dans une prochaine innovation, nous essaierons de donner un enjeu au mesurage et au traitement des mesures, de façon à faire percevoir leur rôle d'outil de décision et de communication.

\section{BIBLIOGRAPHIE}

BEVINGTON, P.R., 1969. Data reduction and error analysis for the physical sciences. (McGraw Hill: New York).

FOUREZ, G., 1988. La construction des sciences. Introduction à la philosophie et à l'éthique des sciences. (DeBoeck Univers:té: Bruxelles).
GIL,D. y CARRASCOSA, J., 1985. Science leaming as a conceptual and methodological change, European Journal of Scientific Education, 7, pp. 231-236.

JOHSUA, S. y DUPIN, J.J., 1989. Représentationset modélisations. le débat scientifique dans la classe et l'apprentisage de la physique. (Berne: Peter Lang). 
KUHN, D., AMSEL, D. y O'LONGHLIM, M., 1988. The development of scientific thinking skills. (Academic Press: New York).

LAROCHELLE, M. y DESAUTELS, J., 1991. Of course it' just obvious! Adolescents'ideas of scientific knowledge, International Journal of Science education, 13(4), pp. 373389.

LAROCHELLE, M. y DESAUTELS, J., 1992. Autour de l'idée de science. Itinéraires cognitifs d'étudiants et étudiantes. (De Boeck-Wesmael: Bruxelles)

LEMEIGNAN, G. y WEIL-BARAIS, A,, 1989. Enseignement et apprentissage $d^{+}$un concept par les élèves: la quantité de mouvement en classe de seconde, Bulletin de l'Union des Physiciens, 716, pp. 1013-1031
MCFARLAND, E., MAC KENZIE, I. y STONE, R., 1987. An undergradiate experiment on statistics and errors using a radioactive source and a microcomputer, American Journal of Physics, 55(7), pp. 650-653.

PETERSON, R.D., 1989. A finite difference technique for propagatin 3 uncertainty in calculations, American Journal of Physics, 57 (10), pp. 925-930.

REED, B.C., 1989. Linear least squares fits with errors in both coordinates, American Journal of Physics, 57(7), pp. 642646

SÉRÉ, M.G.,LARCHER, C. y JOURNEAUX, R., 1992. Learning the statistical analysis of measurements errors, International Journal of Science education, 15(4), pp. 427-438. 